\title{
Cord blood angiogenic progenitor cells are decreased in bronchopulmonary dysplasia
}

\author{
Christopher D. Baker*,\#, Vivek Balasubramaniam*,\#, Peter M. Mourani**\#, \\ Marci K. Sontag*, Claudine P. Black*, Sharon L. Ryan* and Steven H. Abman**
}

ABSTRACT: Bronchopulmonary dysplasia (BPD), the chronic lung disease of prematurity, is associated with impaired vascular and alveolar growth. Antenatal factors contribute to the risk for developing BPD by unclear mechanisms. Endothelial progenitor cells, such as angiogenic circulating progenitor cells (CPCs) and late-outgrowth endothelial colony-forming cells (ECFCs), may contribute to angiogenesis in the developing lung. We hypothesise that cord blood angiogenic CPCs and ECFCs are decreased in preterm infants with moderate and severe BPD.

We quantified ECFCs and the CPC/nonangiogenic-CPC ratio (CPC/non-CPC) in cord blood samples from 62 preterm infants and assessed their relationships to maternal and perinatal risk factors as well as BPD severity. The CPC/non-CPC ratio and ECFC number were compared between preterm infants with mild or no BPD and those with moderate or severe BPD.

ECFC number $(p<0.001)$ and CPC/non-CPC ratio $(p<0.05)$ were significantly decreased in cord blood samples of preterm infants who subsequently developed moderate or severe BPD. Gestational age and birth weight were not associated with either angiogenic marker.

Circulating vascular progenitor cells are decreased in the cord blood of preterm infants who develop moderate and severe BPD. These findings suggest that prenatal factors contribute to late respiratory outcomes in preterm infants.

KEYWORDS: Bronchopulmonary dysplasia, endothelial progenitor cells, neonatal lung disease, prematurity, stem cells

B ronchopulmonary dysplasia (BPD), the chronic lung disease of prematurity, was originally described as the result of postnatal lung injury from mechanical ventilation and oxygen supplementation [1]. While advances in the treatment of neonatal respiratory distress syndrome have improved the survival of extremely low birth weight infants, BPD remains the most common complication of preterm birth [2]. In the post-surfactant era, the "new BPD" is characterised by a developmental arrest of lung vascular and alveolar growth resulting in a decreased surface area for gas exchange [2-4]. BPD is associated with increased mortality and morbidities such as respiratory insufficiency, chronic hypoxaemia, pulmonary hypertension, exercise intolerance, wheezing and poor neurodevelopmental outcomes [5].

The extent of chronic lung disease is highly variable among preterm infants. While gestational age and birth weight are the most predictive indicators of BPD severity, antenatal factors such as pre-eclampsia, chorioamnionitis and intrauterine growth restriction
(IUGR) contribute significantly to the pathogenesis of new BPD $[2,6]$. The Barker Hypothesis suggests that intrauterine factors contribute to the risk of respiratory disease later in life [7]. However, little is known about the underlying mechanisms by which antenatal events contribute to impaired lung development and function. Post-natal factors, such as high levels of supplemental oxygen, patent ductus arteriosus, infection, prolonged mechanical ventilation and malnutrition, are also associated with more severe respiratory outcomes [8]. A subset of infants develops BPD, but the presence or absence of post-natal factors alone may not be sufficient to determine the risk of chronic lung disease in preterm infants.

Both human and animal studies suggest that impaired vascular growth plays a central role in the pathogenesis of $\operatorname{BPD}[3,9,10]$. Vascular endothelial growth factor is decreased in the lungs of human infants dying from severe BPD [9]. Antiangiogenic mediators such as endostatin have been associated with the development of BPD [11].
AFFILIATIONS

*Paediatric Heart Lung Center, University of Colorado School of Medicine, and

\#Children's Hospital Colorado, Aurora, CO, USA.

CORRESPONDENCE

C.D. Baker

Paediatric Heart Lung Center University of Colorado School of Medicine

13123 E. 16th Avenue Box B-395 Aurora CO 80045 USA E-mail: christopher.baker@ ucdenver.edu

Received: Jan 272012 Accepted after revision: March 062012 First published online: April 102012 
In pre-eclampsia, a known contributor to BPD risk, soluble Flt1 (another potent anti-angiogenic factor) is increased [12]. Vascular growth occurs by either angiogenesis (the direct extension of existing vessels) or vasculogenesis [13]. In vasculogenesis, new vessels are formed from primitive haemangioblasts located within a developing organ or from putative bone marrowderived circulating endothelial progenitor cells (EPCs) that are thought to home to developing microcapillary beds in the lung and other organ systems $[13,14]$.

EPCs are decreased in the blood, lungs and bone marrow of newborn mice with experimental BPD due to hyperoxia [15]. This suggests that post-natal events can disrupt vasculogenic mechanisms during development leading to impaired lung vascular and alveolar growth. Systemic and intra-tracheal administration of mesenchymal stromal cells (MSCs) or MSCconditioned media to neonatal rodents partially restores lung architecture during neonatal hyperoxia [16, 17]. In addition, intravenous administration of bone marrow-derived proangiogenic cells after exposure to neonatal hyperoxia normalises lung structure in infant mice [18].

Based on these data from preclinical models of BPD, we speculate that angiogenic progenitor levels in umbilical cord blood samples at birth may be inversely associated with the severity of BPD. More specifically, we hypothesise that preterm infants who have decreased levels of angiogenic progenitors in their cord blood will have an increased risk of developing severe $\mathrm{BPD}$. In this prospective cohort study, we report that preterm infants who later develop moderate and severe BPD have decreased angiogenic progenitor cells in umbilical cord blood, as reflected by reduced late-outgrowth endothelial colonyforming cells (ECFCs) and by the ratio of pro-angiogenic circulating progenitor cells (CPCs) to nonangiogenic CPCs (CPC/non-CPC).

\section{METHODS}

\section{Subject enrolment and data collection}

The Colorado Multiple Institutional Review Board (Aurora, CO, USA) approved all study protocols. Informed consent was obtained from pregnant mothers presenting in preterm labour. Subjects were enrolled from October 2009 until June 2011. Cord blood was collected and processed as previously described [19]. Eligible mothers were admitted to the University Hospital Anschutz Inpatient Pavilion (Aurora, CO), delivering a newborn of 24-36 weeks gestational age and capable of providing consent. Exclusion criteria included known HIV, or hepatitis B or $\mathrm{C}$ infection. Maternal and infant clinical data were collected until hospital discharge. A clinical diagnosis of chorioamnionitis was made in the setting of uterine tenderness and maternal fever. IUGR was defined as birth weight less than the 10th percentile with an occipitofrontal circumference greater than the 10th percentile. Data were stored in a secure REDCap database [20].

\section{Determination of BPD status}

The presence and severity of BPD were determined using US National Institutes of Health criteria with an adjustment for the local altitude of $1,600 \mathrm{~m}$ (fig. S1) [8]. An oxygen reduction test (online supplementary material) was performed when indicated. Briefly, the diagnosis of BPD was made at 36 weeks postconception (or day of life 28 if the infant was born after
32 weeks). Infants who required supplemental oxygen for $<28$ days had no BPD. Those infants with an inspiratory oxygen fraction $\left(F \mathrm{I}, \mathrm{O}_{2}\right)$ of $<0.26$ had mild BPD. If the $\mathrm{FI}_{1} \mathrm{O}_{2}$ was between 0.26 and 0.35 , the infant had moderate BPD. Finally, severe BPD indicates that the child required an $\mathrm{FI}, \mathrm{O}_{2}$ of $>0.35$ or positive pressure (mechanical ventilation, continuous positive airway pressure (CPAP) or high-flow oxygen by nasal cannula). Subjects were separated into two study groups, those with mild or no BPD and those with moderate or severe BPD, based on the rationale that at sea-level infants with mild or no BPD do not require supplemental oxygen at the time of diagnosis.

\section{Cord blood collection and ECFC isolation}

Samples were maintained at room temperature and analysed within $24 \mathrm{~h}$. Mononuclear cells (MNCs) were isolated by gradient centrifugation for both the ECFC culture assay and polychromatic flow cytometry (PFC). MNCs were cultured on type 1 collagen in complete EGM-2 media (Lonza, Basel, Switzerland) with $10 \%$ fetal bovine serum. ECFC colonies (fig. S2a) were identified daily and enumerated on day 14. Low-passage (P2-3) ECFCs were characterised by immunohistochemistry, PFC, tube formation and a single-cell assay to confirm self-renewal (fig. S2b-e).

\section{Polychromatic flow cytometry}

MNCs (fig. S3a) were analysed by PFC as follows. 0.5-1.0 $\times 10^{6}$ cells were stained with antibodies to CD31, CD34, CD45, AC133, glycophorin-A (erythrocyte exclusion) and CD14 (macrophage exclusion), and the LIVE/DEAD ${ }_{\circledast}$ Cell Viability Assay (Life Technologies, Grand Island, NY, USA). Compensation beads, fluorescence minus-one controls and bi-exponential gating were utilised to facilitate accurate compensation and gating [21]. Angiogenic (CD45-dim CD34+ CD31+ AC133+) and nonangiogenic (CD45-dim CD34+ CD31+ AC133-) CPCs were measured so that the $\mathrm{CPC} /$ non-CPC ratio could be determined using established methods (fig. S3b-c) [21, 22]. PFC analysis was performed using a CyAn nine-color flow cytometer (Beckman Coulter, Brea, CA, USA) and FlowJo software (version 9.3.2; Beckman Coulter).

\section{Statistical analysis}

Nonparametric data were analysed using Mann-Whitney tests and are presented as medians with interquartile ranges (IQRs). Normally distributed data were analysed using unpaired t-tests and are presented as means with standard deviations. Spearman's correlation coefficients were utilised to compare ECFC number with gestational age and birth weight. Fisher's exact test was used to analyse categorical data. Analysis was performed with the Prism software package (version 5.0; GraphPad, La Jolla, CA, USA). The significance level was set at $\alpha=0.05$. C.D. Baker and a biostatistician (M.K. Sontag) analysed all data.

\section{RESULTS}

\section{Patient characteristics}

As summarised in table 1, 62 preterm infants (24-36 weeks gestational age) were enrolled. $13(21.0 \%)$ developed moderate or severe BPD. The remaining $49(79.0 \%)$ developed no or mild BPD. In comparison with infants who had mild or no BPD, gestational age, birth weight, length, and head circumference were significantly decreased in patients with moderate or severe BPD (table 1). Ventilator days, CPAP days, CPAP plus 


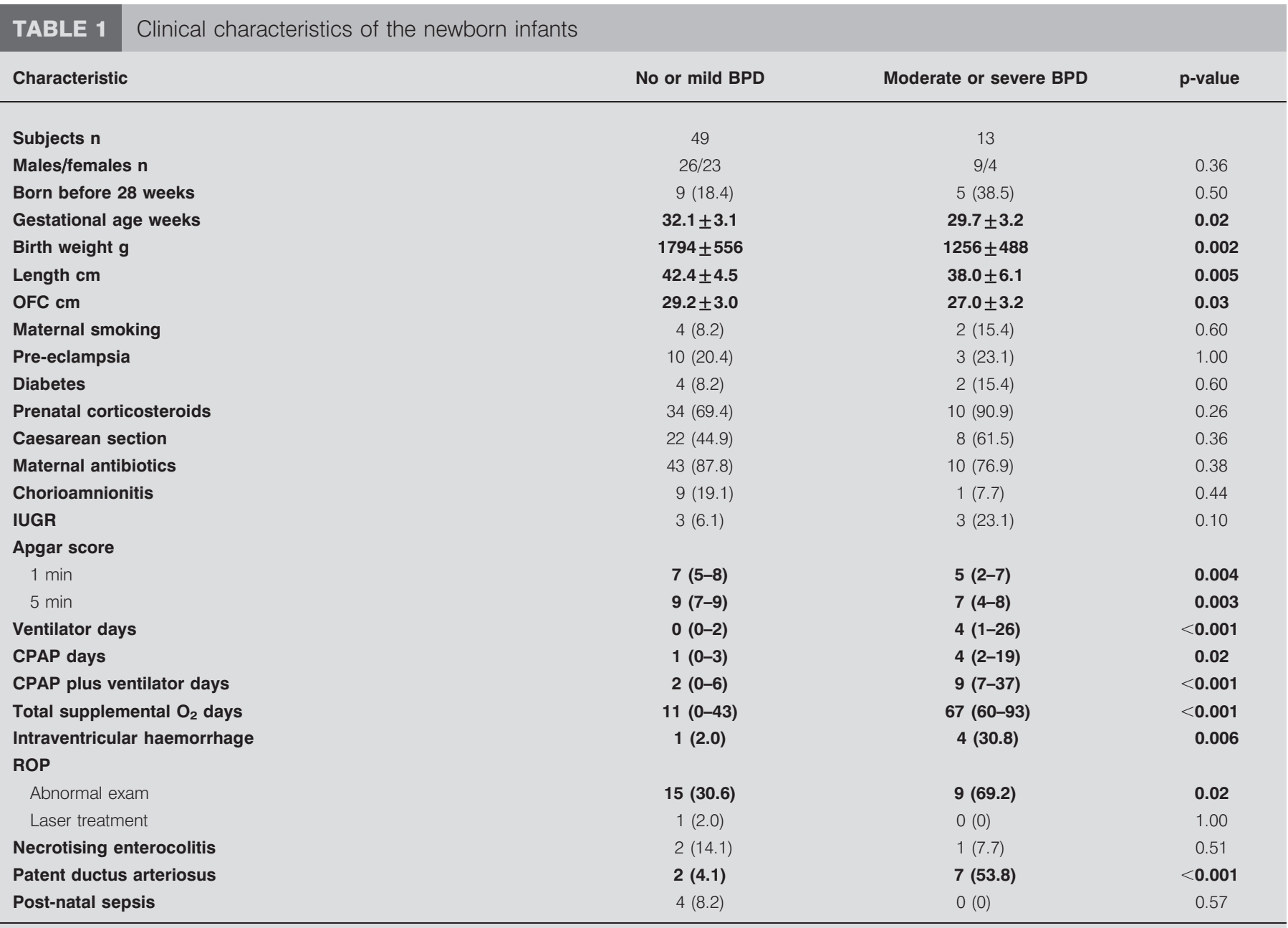

Data are presented as $n(\%)$, mean \pm SD or median (interquartile range), unless otherwise stated. Bold indicates statistical significance. BPD: bronchopulmonary dysplasia; OFC: occipitofrontal circumference; IUGR: intrauterine growth restriction; CPAP: continuous positive airway pressure; ROP: retinopathy of prematurity

ventilator days and total days with supplemental oxygen were greater in the more severe group. The incidences of preeclampsia, chorioamnionitis, maternal diabetes and smoking were similar between groups. Apgar scores at 1 and $5 \mathrm{~min}$ were greater in the mild or no BPD group.

\section{Angiogenic circulating progenitor cells are decreased in moderate and severe BPD}

PFC was performed on the cord blood of 60 of the 62 subjects. 12 samples were excluded because fluorochrome oversaturation prevented CPC enumeration. Of the remaining 48, 37 had mild or no BPD and 11 had moderate or severe BPD. The CPC/non$\mathrm{CPC}$ ratio was significantly lower in the cord blood of infants who developed moderate or severe BPD (1.5, IQR 1.2-1.8 versus 2.5, IQR 1.5-3.9; $\mathrm{p}<0.05$ ) (fig. 1a). When samples from all infants who later developed BPD $(n=29)$ were compared with those who did not $(n=19)$, there was a trend for the CPC/non-CPC ratio to be lower in those with $\mathrm{BPD}$, but this failed to achieve statistical significance (1.8, IQR 1.3-3.0 versus 2.8, IQR 1.8-4.9; $\mathrm{p}=0.07$ ) (fig. S4a). In the subset of infants born at or before 28 weeks $(n=11)$, the trend towards a decreased CPC/non-CPC ratio was not statistically significant (fig. S4b).

\section{Cord blood ECFCs are decreased in moderate and severe BPD}

Cord blood ECFC number was markedly decreased in preterm infants who went on to develop moderate or severe BPD as compared with those who did not (0, IQR $0-0.8$ versus 3.6, IQR $0.9-8.0$ colonies per $10^{7} \mathrm{MNCs}$; $<<0.001$ ) (fig. 1b). ECFCs were also decreased among all subjects with $\operatorname{BPD}(n=36)$ as compared with those without BPD ( $n=26)(1.4$, IQR $0-4.8$ versus 3.9 , IQR 1.3-7.4 colonies per $10^{7} \mathrm{MNCs} ; \mathrm{p}<0.05$ ) (fig. 1c). Among infants born at or before 28 weeks, all developed BPD. However, none of the infants with moderate or severe BPD had detectable ECFCs in their cord blood. Infants with mild BPD had 4.9 (IQR 2.4-9.0) ECFC colonies per $10^{7}$ MNCs (p<0.01) (fig. 1d).

By univariate nonparametric comparison, neither gestational age $(r=0.23, p=0.11)$ nor birth weight $(r=0.24, p=0.11)$ affected the $\mathrm{CPC} /$ non-CPC ratio. In contrast to previous studies [23, $24]$, we found that gestational age did not correlate with cord blood ECFC number $(r=0.17, p=0.18$; fig. $2 a)$. Although a nonparametric comparison of ECFC number with birth weight was statistically significant, the coefficient of determination shows that the correlation is small $(r=0.26, p=0.04)$ (fig. $54 c)$. 

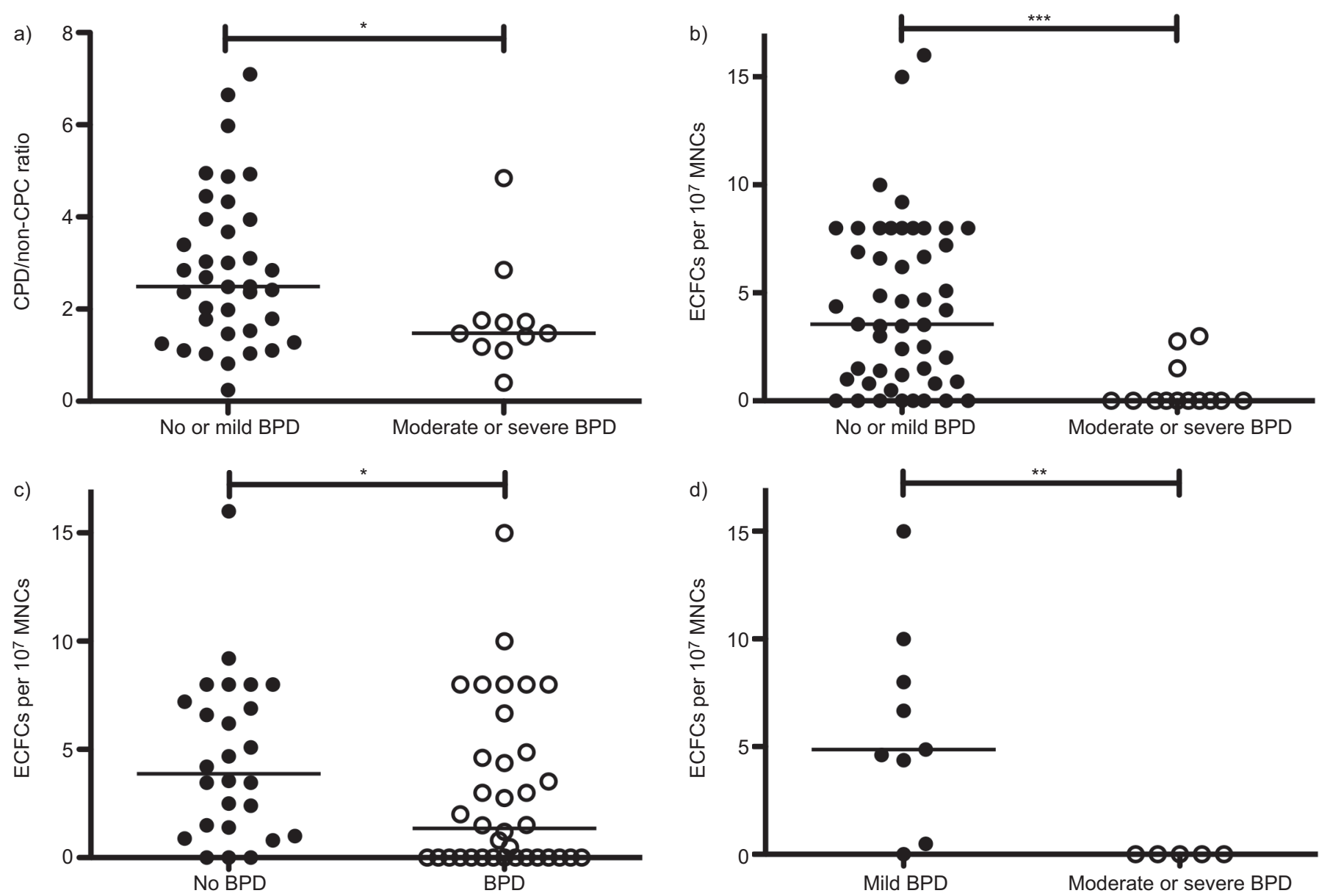

FIGURE 1. Angiogenic progenitor levels in preterm newborns by bronchopulmonary dysplasia (BPD) severity. a) The ratio of angiogenic circulating progenitor cells (CPCs) to nonangiogenic CPCs (CPC/non-CPC) was significantly greater in the cord blood of infants with mild or no BPD $(n=37)$ as compared with those with moderate or severe BPD ( $n=11)$. b) Cord blood endothelial colony-forming cell (ECFC) number, defined as the number of ECFC colonies on day 14 per $10^{7}$ mononuclear cells (MNCs) plated, was significantly greater in infants who later developed mild or no BPD $(n=49)$ as compared with those infants who developed moderate or severe BPD ( $n=13)$. c) Cord blood ECFC number was greater in infants with no BPD $(n=26)$ as compared with those with BPD of any severity ( $n=36)$. d) Among infants born at $\leqslant 28$ weeks gestational age, cord blood ECFC number was significantly decreased in infants with moderate or severe BPD ( $n=5)$ as compared with those with mild BPD ( $n=9$ ). ${ }^{*}: p<0.05 ;{ }^{* *}: p<0.01 ;{ }^{* * *}: p<0.001$.

A small but significant correlation was found between the CPC/ non-CPC ratio and ECFC number $(r=0.33$, $p=0.02)$ (fig. $S 4 d$ ). We then compared samples that contained ECFCs $(n=32)$ to those that did not $(n=16)$ and found that the $C P C /$ non-CPC ratio was significantly higher in ECFC-containing cord blood (2.8, IQR 1.8-4.2 versus 1.5, IQR 1.1-1.8; $\mathrm{p}=0.001$ ) (fig. $2 \mathrm{~b}$ ).

\section{Association of perinatal risk factors and cord blood ECFC number}

Although its incidence was no different between groups, we found that infants with clinical chorioamnionitis $(n=10)$ had increased cord blood ECFCs (8.0, IQR 3.9-8.5 versus 1.8, IQR 04.7 colonies per $10^{7} \mathrm{MNCs} ; \mathrm{p}<0.01$ ) (fig. 3a). In the present study, cord blood ECFCs tended to be lower in maternal preeclampsia $(n=13)$, but the difference was not statistically significant (1.0, IQR 0-4.7 versus 3.0, 0-7.0 colonies per $10^{7}$ MNCs; $p=0.23$ ) (fig. S4e). Birth by Caesarean section $(n=30)$ resulted in a significantly decreased cord blood ECFC number as compared with vaginal birth (1.1, IQR 0-3.5 versus 5.0 , IQR $1.0-8.0$ colonies per $10^{7} \mathrm{MNCs} ; \mathrm{p}<0.01$ ) (fig. $3 \mathrm{~b}$ ). There was no difference between planned and emergent Caesarean delivery (data not shown). Antenatal corticosteroid treatment did not alter ECFC number (fig. S4f). None of these antenatal factors significantly affected the $\mathrm{CPC} /$ non-CPC ratio.

\section{DISCUSSION}

We report that circulating angiogenic progenitor cells, including late-outgrowth ECFCs and the $\mathrm{CPC} /$ non-CPC ratio, are decreased in the cord blood of preterm infants who develop moderate or severe BPD. In this population, neither the CPC/ non-CPC ratio nor ECFC numbers are strongly associated with prematurity, as determined by gestational age or birth weight. Reduction in both angiogenic markers was associated with increased BPD. Preterm newborns with chorioamnionitis have increased cord blood ECFCs and infants delivered via Caesarean section have decreased ECFCs. While enrolment was not sufficient to demonstrate a significant difference, preterm infants born to mothers with pre-eclampsia tended to have decreased cord blood ECFCs. 

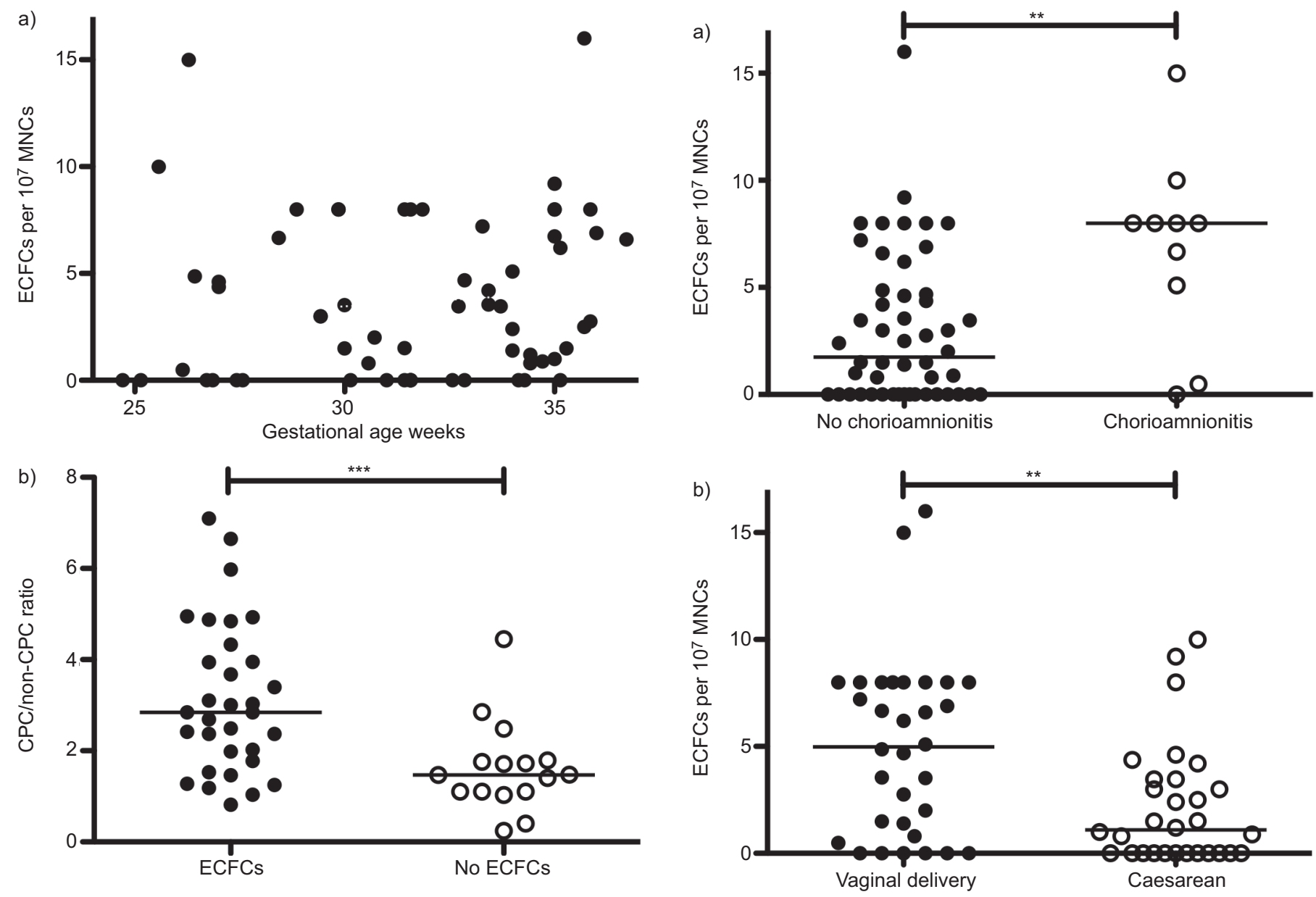

FIGURE 2. Cord blood endothelial colony-forming cell (ECFC) number (on day 14) plotted against gestational age and the ratio of angiogenic circulating progenitor cells (CPCs) to nonangiogenic CPCs (CPC/non-CPC). a) There was no correlation between cord blood ECFC number and gestational age (Spearman's $r=0.17, p=0.18)$. b) In infants whose cord blood yielded no ECFC colonies, the $\mathrm{CPC} /$ non-CPC ratio was significantly decreased as compared with those with cord blood ECFCs. MNC: mononuclear cell. ***: $p<0.001$.

A recent epidemiological study supported Barker's Hypothesis by demonstrating links between antenatal events and late respiratory outcomes such as BPD [6]. However, HANSEN et al. [6] noted that only $54 \%$ of the odds variability for developing BPD could be described using the occurrence of pre-eclampsia, clinical chorioamnionitis, male sex and maternal smoking as well as gestational age and birth weight z-score. Our findings are interesting in that cord blood ECFC and CPC/non-CPC levels are low in preterm infants who subsequently develop moderate or severe BPD. These data directly support the concept that antenatal events affect late respiratory outcomes in preterm infants, and suggest that EPCs may serve as potential biomarkers to better identify at risk infants. However, whether altered EPC levels or function directly contribute to the pathobiology of BPD remains unknown.

Although first isolated $>10$ yrs ago, much remains unknown about the role of EPCs in vascular growth during fetal development. EPCs have been isolated and quantified by both PFC and primary culture assays [25, 26]. Cultured EPCs are

FIGURE 3. The influence of prenatal factors on cord blood endothelial colonyforming cell (ECFC) number. a) ECFCs were increased in the cord blood of infants born with clinical chorioamnionitis $(n=10)$ as compared with those without chorioamnionitis $(n=50)$. b) Infants born via Caesarean section $(n=30)$ had significantly fewer cord blood ECFCs than those born vaginally $(n=32)$. MNC: mononuclear cell. ${ }^{* *}: \mathrm{p}<0.01$.

increased in human disease states such as acute lung injury in which early-outgrowth EPCs correlate with survival [27]. Although early-outgrowth EPCs augment angiogenesis, these cells do not have an endothelial morphology and function as angiogenic macrophages [28, 29]. In comparison, late-outgrowth ECFCs have an endothelial appearance, are stem cell-like (highly proliferative and self-renewing) and form chimaeric vessels in vivo $[26,29]$. However, ECFCs may have fewer paracrine effects on angiogenesis [28]. Both early EPCs and ECFCs may be required for effective angiogenesis in the developing lung.

EPCs were first described by PFC as CD34+ AC133+ KDR+ cells $[14,30]$. However, we did not measure these cells as they are not different in the cord blood of full-term infants and preterm infants whether or not they later develop BPD [19, 31]. In contrast, CPCs express a different surface antigen profile (CD45dim CD34+ CD31+ AC133+), are proangiogenic and promote tumour growth in vivo [22]. Therefore, we included a CD31 antibody in our staining protocol in lieu of identifying KDR+ cells. Non-CPCs are AC133- (CD45-dim CD34+ CD31+ AC133-) and are not angiogenic [22]. The $\mathrm{CPC} /$ non-CPC ratio is 
decreased in peripheral artery disease and gestational diabetes, but its significance in the cord blood of preterm infants with BPD has not been previously described [22,32]. While the absolute number of circulating CPCs may be relevant, there was no difference in CPCs between study groups. The CPC/non-CPC ratio may more accurately reflect the balance between competing mechanisms the body uses to regulate vascular growth.

A previous study suggested that ECFCs may be decreased in a small subset of patients with BPD, but whether these findings simply reflect the association between the severity of BPD and degree of prematurity was unclear [23]. We did not observe ECFC number to be a function of gestational age or birth weight, as previously reported in two smaller studies, suggesting that the link between ECFCs and late outcomes are not simply due to an association with the degree of prematurity [23, 24]. Circulating progenitors may directly contribute to pulmonary angiogenesis [10, 15, 18], and we speculate that decreased ECFCs may contribute to abnormal vascular growth and more severe BPD. Further study is needed to better elucidate the role of CPCs and ECFCs during normal vascular development and how changes in EPC number or function contribute to BPD.

Cord blood ECFCs are increased with chorioamnionitis and vaginal birth. We speculate that these processes result in significant perinatal stress that causes a release of angiogenic progenitors into the circulation. We note that the $\mathrm{CPC} /$ nonCPC ratio was not similarly increased in these infants. Further study is needed to confirm these findings and to identify the angiogenic factors that mediate this response.

The small number of patients enrolled in the study may result in type II errors and limits our ability to perform multivariate analysis in depth. These findings neither confirm nor refute the hypothesis that decreased circulating EPCs actually contribute to impaired vascular growth in BPD. Nevertheless, the striking association between decreased EPCs and BPD severity leads us to speculate that disruption of pro-angiogenic precursor cells are involved in the pathogenesis of BPD.

We conclude that angiogenic CPCs and ECFCs, potential cellular biomarkers of angiogenic activity, are decreased in the cord blood of preterm infants who go on to develop moderate or severe BPD. Using the data presented, we determined that a $\mathrm{CPC} /$ non-CPC ratio of $<2$ and the absence of cord blood ECFCs convey relative risks of 5.2 (95\% CI 1.2-21.9) and 8.1 (95\% CI 2.526.2), respectively. However, further large-scale trials including validation cohorts are needed to confirm whether either of these assays provides biomarkers that predict which infants are at greatest risk for chronic lung disease. We speculate that antenatal factors, genetic predisposition, or both, decrease circulating EPCs in the fetal circulation, which may impair post-natal vasculogenesis and contribute to the severity of late respiratory outcomes after preterm birth. A better understanding of the function of both CPCs and ECFCs will lead to novel therapies to promote pulmonary vascular growth in the preterm newborn and improve outcomes in this high-risk neonatal population.

\section{SUPPORT STATEMENT}

This study was supported by grants from the US National Institutes of Health (K12 HL090147-01 to C.D. Baker, R01 HL089262 to V. Balasubramaniam, NCRR 5K23RR021921 to P.M. Mourani, HL085703-01 and R01 HL68702 to S.H. Abman) and Colorado CTSI 5UL1 RR025780 and the Thrasher Foundation (to S.H. Abman).

\section{STATEMENT OF INTEREST}

None declared.

\section{ACKNOWLEDGEMENTS}

The authors would like to thank L. Fashaw (Perinatal Clinical Translational Research Center, Children's Hospital Colorado, Aurora, CO USA) for study coordination, D. Ingram, M. Yoder (both Indiana University, Indianapolis, IN, USA), J. Gien and C. White for their ongoing collaboration, and insight and B. Wisniewski and G. Seedorf (all Paediatric Heart Lung Center, University of Colorado School of Medicine, Aurora, CO) for technical support.

\section{REFERENCES}

1 Northway WH, Rosan RC, Porter DY. Pulmonary disease following respirator therapy of hyaline-membrane disease. Bronchopulmonary dysplasia. N Engl J Med 1967; 276: 357-368.

2 Jobe AH. The new bronchopulmonary dysplasia. Curr Opin Pediatr 2011; 23: 167-172.

3 Abman SH. Bronchopulmonary dysplasia: "a vascular hypothesis". Am J Respir Crit Care Med 2001; 164: 1755-1756.

4 Coalson JJ, Winter VT, Siler-Khodr T, et al. Neonatal chronic lung disease in extremely immature baboons. Am J Respir Crit Care Med 1999; 160: 1333-1346.

5 Baraldi E, Filippone M. Chronic lung disease after premature birth. N Engl J Med 2007; 357: 1946-1955.

6 Hansen AR, Barnés CM, Folkman J, et al. Maternal preeclampsia predicts the development of bronchopulmonary dysplasia. J Pediatr 2010; 156: 532-536.

7 Barker DJ. The intrauterine origins of cardiovascular and obstructive lung disease in adult life. The Marc Daniels Lecture 1990. J R Coll Physicians Lond 1991; 25: 129-133.

8 Jobe AH, Bancalari E. Bronchopulmonary dysplasia. Am J Respir Crit Care Med 2001; 163: 1723-1729.

9 Bhatt AJ, Pryhuber GS, Huyck H, et al. Disrupted pulmonary vasculature and decreased vascular endothelial growth factor, Flt-1, and TIE-2 in human infants dying with bronchopulmonary dysplasia. Am J Respir Crit Care Med 2001; 164: 1971-1980.

10 Jakkula M, Le Cras TD, Gebb S, et al. Inhibition of angiogenesis decreases alveolarization in the developing rat lung. Am J Physiol Lung Cell Mol Physiol 2000; 279: L600-L607.

11 Janer J, Andersson S, Kajantie E, et al. Endostatin concentration in cord plasma predicts the development of bronchopulmonary dysplasia in very low birth weight infants. Pediatrics 2009; 123: 1142-1146.

12 Maynard SE. Excess placental soluble fms-like tyrosine kinase 1 (sFlt1) may contribute to endothelial dysfunction, hypertension, and proteinuria in preeclampsia. J Clin Invest 2003; 111: 649-658.

13 Flamme I, Frölich T, Risau W. Molecular mechanisms of vasculogenesis and embryonic angiogenesis. J Cell Physiol 1997; 173: 206-210.

14 Asahara T, Murohara T, Sullivan A, et al. Isolation of putative progenitor endothelial cells for angiogenesis. Science 1997; 275: 964-967.

15 Balasubramaniam V, Mervis CF, Maxey AM, et al. Hyperoxia reduces bone marrow, circulating, and lung endothelial progenitor cells in the developing lung: implications for the pathogenesis of bronchopulmonary dysplasia. Am J Physiol Lung Cell Mol Physiol 2007; 292: L1073-L1084.

16 Aslam M, Baveja R, Liang OD, et al. Bone marrow stromal cells attenuate lung injury in a murine model of neonatal chronic lung disease. Am J Respir Crit Care Med 2009; 180: 1122-1130. 
17 Van Haaften T, Byrne R, Bonnet S, et al. Airway delivery of mesenchymal stem cells prevents arrested alveolar growth in neonatal lung injury in rats. Am J Respir Crit Care Med 2009; $1-55$.

18 Balasubramaniam V, Ryan SL, Seedorf GJ, et al. Bone marrowderived angiogenic cells restore lung alveolar and vascular structure after neonatal hyperoxia in infant mice. Am J Physiol Lung Cell Mol Physiol 2010; 298: L315-L323.

19 Baker CD, Ryan SL, Ingram DA, et al. Endothelial colonyforming cells from preterm infants are increased and more susceptible to hyperoxia. Am J Respir Crit Care Med 2009; 180: 454-461.

20 Harris PA, Taylor R, Thielke R, et al. Research electronic data capture (REDCap) - a metadata-driven methodology and workflow process for providing translational research informatics support. J Biomed Inform 2009; 42: 377-381.

21 Estes ML, Mund JA, Ingram DA, et al. Identification of endothelial cells and progenitor cell subsets in human peripheral blood. Curr Protoc Cytom 2010; 9: 1-11.

22 Estes ML, Mund JA, Mead LE, et al. Application of polychromatic flow cytometry to identify novel subsets of circulating cells with angiogenic potential. Cytometry A 2010; 77: 831-839.

23 Borghesi A, Massa M, Campanelli R, et al. Circulating endothelial progenitor cells in preterm infants with bronchopulmonary dysplasia. Am J Respir Crit Care Med 2009; 180: 540-546.

24 Ligi I, Simoncini S, Tellier E, et al. A switch toward angiostatic gene expression impairs the angiogenic properties of endothelial progenitor cells in low birth weight preterm infants. Blood 2011; 118: 1699-1709.

25 Hill JM, Zalos G, Halcox JP, et al. Circulating endothelial progenitor cells, vascular function, and cardiovascular risk. N Engl J Med 2003; 348: 593-600.

26 Ingram DA, Mead LE, Tanaka H, et al. Identification of a novel hierarchy of endothelial progenitor cells using human peripheral and umbilical cord blood. Blood 2004; 104: 2752-2760.

27 Burnham EL, Taylor WR, Quyyumi AA, et al. Increased circulating endothelial progenitor cells are associated with survival in acute lung injury. Am J Respir Crit Care Med 2005; 172: 854-860.

28 Sieveking DP, Buckle A, Celermajer DS, et al. Strikingly different angiogenic properties of endothelial progenitor cell subpopulations: insights from a novel human angiogenesis assay. J Am Coll Cardiol 2008; 51: 660-668.

29 Yoder MC, Mead LE, Prater D, et al. Redefining endothelial progenitor cells via clonal analysis and hematopoietic stem/ progenitor cell principals. Blood 2007; 109: 1801-1809.

30 Peichev M, Naiyer AJ, Pereira D, et al. Expression of VEGFR-2 and AC133 by circulating human $\mathrm{CD} 34^{+}$cells identifies a population of functional endothelial precursors. Blood 2000; 95: 952-958.

31 Paviotti G, Fadini GP, Boscaro E, et al. Endothelial progenitor cells, bronchopulmonary dysplasia and other short-term outcomes of extremely preterm birth. Early Hum Dev 2011; 87: 461-465.

32 Acosta JC, Haas DM, Saha CK, et al. Gestational diabetes mellitus alters maternal and neonatal circulating endothelial progenitor cell subsets. Am J Obstet Gynecol 2011; 204: 254.e258-254.e215. 